\title{
1 Modeling human age-associated increase in Gadd45y expression leads to spatial \\ 2 recognition memory impairments in young adult mice
}

3 David V.C. Brito ${ }^{1}$, Kubra Gulmez Karaca ${ }^{1,3}$, Janina Kupke ${ }^{1}$, Franziska Mudlaff ${ }^{1}$, Benjamin

4 Zeuch $^{1,4}$, Rui Gomes ${ }^{2}$, Luísa V. Lopes², Ana M.M. Oliveira ${ }^{1 *}$

5

$6{ }^{1}$ Department of Neurobiology, Interdisciplinary Centre for Neurosciences (IZN), Heidelberg

7 University, INF 366, 69120 Heidelberg, Germany

$8{ }^{2}$ Instituto de Medicina Molecular João Lobo Antunes, Faculdade de Medicina de Lisboa,

9 Universidade de Lisboa, 1649-028 Lisbon, Portugal

$10{ }^{3}$ Present address: Department of Cognitive Neuroscience, Donders Institute for Brain,

11 Cognition and Behaviour, Radboud University Medical Center Kapittelweg 29, 6525 EN

12 Nijmegen, The Netherlands

$13{ }^{4}$ Present address: Directors' Research, European Molecular Biology Laboratory (EMBL),

14 Meyerhofstrasse 1, 69117 Heidelberg, Germany

$16{ }^{*}$ Corresponding author:

17 Ana MM Oliveira

18 Department of Neurobiology, Interdisciplinary Center for Neurosciences (IZN)

19 Heidelberg University

$20 \quad$ Im Neuenheimer Feld 366

2169120 Heidelberg, Germany

22 Telephone: +4962215416510

23 Fax: +4962215416524

24 E-mail: oliveira@nbio.uni-heidelberg.de 
Abstract

Aging is associated with the progressive decay of cognitive function. Hippocampus-

dependent processes, such as the formation of spatial memory, are particularly vulnerable to aging. Currently, the molecular mechanisms responsible for age-dependent cognitive decline are largely unknown. Here, we investigated the expression and function of the growth arrest DNA damage gamma (Gadd45y) during aging and cognition. We report that Gadd45ץ expression is increased in the hippocampus of aged humans and that Gadd45y overexpression in the young adult mouse hippocampus compromises cognition. Moreover, Gadd45y overexpression in hippocampal neurons disrupted CREB signaling and the expression of well-established activity-regulated genes. This work shows that Gadd45y expression is tightly controlled in the hippocampus and its disruption may be a mechanism contributing to age-related cognitive impairments observed in humans.

Keywords: Activity-regulated gene expression, age-related cognitive deficits, CREB,

40 Gadd45y, object location memory. 


\section{Introduction}

52 Age-related cognitive decline in humans affects about $40 \%$ of individuals aged 65 years or older (Aigbogun et al., 2017), even though deterioration of cognitive functions may start earlier (Singh-Manoux et al., 2012). Long-term memory formation requires activity-regulated signaling that results in de novo gene expression. These genomic responses are known to be disrupted in the aged hippocampus (Stefanelli et al., 2018). Therefore, age-associated changes that dysregulate the coupling between neuronal activity and gene transcription likely underlie age-related cognitive deficits.

Recently, we and others showed that the growth arrest DNA damage gamma (Gadd45ץ) is required for memory formation in the prelimbic prefrontal cortex (Li et al., 2018) and the hippocampus (Brito et al., 2020). Moreover, we found that aging reduces Gadd45y expression in the mouse hippocampus and that mimicking this reduction in young adult mice induces age-like memory impairments (Brito et al., 2020). At the molecular level, Gadd45y is required for CAMP response element-binding protein (CREB) activation in response to neuronal activity and associated gene expression (Brito et al., 2020). In the current study, we found that in postmortem human hippocampal tissue from aged individuals, Gadd45 $\gamma$ expression levels are increased relative to young donors. Furthermore, we showed that increasing Gadd45y levels in the mouse hippocampus led to impairments in memory formation and disrupted CREB activation and memory-related gene expression in cultured hippocampal neurons.

71 Overall, this work together with our previous findings (Brito et al., 2020), demonstrate a requirement for tight regulation of neuronal Gadd45y levels in gene expression regulation

73 and cognitive abilities. Thus, dysregulation of Gadd45y expression might be an underlying mechanism involved in age-related cognitive impairments observed in mice and humans.

\section{Materials and Methods}


2.1. Subjects. The use of human samples was conducted in accordance with the Helsinki Declaration as well as national ethical guidelines. Protocols were approved by the Local Ethics Committee and the National Data Protection Committee. The biospecimens were obtained $36 \mathrm{~h}$ postmortem from healthy aged (60-65 years old) and young (21-22 years old) individuals (Temido-Ferreira et al., 2018). The tissue was processed and preserved for molecular analyses as previously described (Pliassova et al., 2016). Young adult male C57BL/6N mice (Charles River, Sulzfeld, Germany) were 3-months-old at the time of behavior experiments. Mice were group-housed (3-4 mice per cage) on a 12h light/dark cycle $\left(22 \pm 1^{\circ} \mathrm{C}, 55 \pm 10 \%\right.$ relative humidity) with ad libitum access to water and food. All behavioral experiments took place during the light phase. All procedures were carried out in accordance with German guidelines for the care and use of laboratory animals and with the European Community Council Directive 86/609/EEC.

\subsection{Recombinant adeno-associated virus (rAAVs). Viral particles were}

produced and purified as described previously (Gulmez Karaca et al., 2020). Overexpression

of Gadd45y was achieved by using a viral vector that contained the mouse CamKIl $\alpha$

promoter upstream of the Gadd45y full-length mouse cDNA sequence. As a control vector, we used a construct containing the CamKIl $\alpha$ promoter driving the expression of GFP. For each virus batch, toxicity was analyzed on primary hippocampal cultures before the start of the experiments. For this, different regions of the coverslip were imaged using identical microscope settings and the number of dead cells was quantified using Fiji (Schindelin et al., 2012) on day in vitro (DIV) 10.

2.3. Primary hippocampal cultures. Hippocampal cultures from newborn C57BI/6N mice (Charles River, Sulzfeld, Germany) were prepared and maintained as previously described (Gulmez Karaca et al., 2020). rAAV infection of cultures occurred on

101 DIV 4. Experiments were performed on DIV 10. To induce action potential bursting, cultures 102 were treated with $50 \mu \mathrm{M}$ bicuculline (Enzo Life Sciences, Germany).

$103 \quad$ 2.4. Stereotaxic surgery. rAAVs were injected into the dorsal hippocampus at the 104 following coordinates relative to Bregma: $-2 \mathrm{~mm}$ anteroposterior, $\pm 1.5 \mathrm{~mm}$ medio-lateral, - 
$1051.7,-1.9$ and $-2.1 \mathrm{~mm}$ dorsoventral. A total volume of $1.5 \mu \mathrm{l}$ was injected per hemisphere

106 at $200 \mathrm{nl} / \mathrm{min}$. Following injections at each individual site, the needle was left in place for $60 \mathrm{~s}$.

107 Behavioral experiments started 2 weeks after rAAVs delivery. After behavioral testing,

108 histological analysis was performed to confirm tissue and cellular integrity.

$109 \quad$ 2.5. Behavioral testing. Before behavioral testing started, mice were habituated

110 to the experimenter and behavioral room by handling for 3 consecutive days, 1 minute per

111 mouse. Object-location test and contextual fear conditioning were performed as previously

112 described (Oliveira et al., 2012; Oliveira et al., 2016). The open field test was carried out

113 within the first session of the object-place recognition training as previously described

114 (Gulmez Karaca et al., 2018).

115 2.6. Quantitative reverse-transcription PCR. Total RNA from human tissue was 116 extracted and cDNA produced as previously described (Temido-Ferreira et al., 2018). For

117 RNA isolation from mouse hippocampal tissue, the tissue was rapidly dissected, placed in

118 RNAlater (Sigma, Munich, Germany) and isolated using the RNeasy Plus Mini Kit (Qiagen,

119 Hilden, Germany) with additional on-column DNase I digestion, according to the

120 manufacturer's instructions. cDNA production and quantitative reverse-transcription PCR (q-

121 RT-PCR) was performed as previously described (Brito et al., 2020). The following TaqMan

122 probes were used: Arc (Mm00479619_g1), c-Fos (Mm00487425_m1), FosB

123 (Mm00500401_m1), Gadd45a (Mm00432802_m1), Gadd45ß (Mm00345123_m1), Gadd45Y

124 (Mm00442225_m1), Egr1 (Mm00656724_m1) and Npas4 (Mm00463644_m1). For human

125 genes the following TaqMan probes were used: Gadd45a (Hs00169255_m1), Gadd45 $\beta$

126 (Hs00169587_m1), Gadd45y (Hs00198672_m1). Expression levels of target genes were

127 normalized to the expression of the housekeeping gene GusB (Mm00446953_m1) or $\beta$-actin

128 (Hs01060665_g1) for mouse or human genes, respectively. Controls were used to exclude

129 the possibility of DNA or RNA contaminations.

$130 \quad$ 2.7. Western blotting. Western blotting was performed as previously described

131 with minor modifications (Brito et al., 2020; Gulmez Karaca et al., 2020). Briefly, hippocampal

132 cultures infected on DIV 4 were lysed on DIV 10 in SDS sample buffer. In the case of 
133 western blotting of tissue samples, the dorsal hippocampus was microdissected from mouse

134 brain and homogenized in RIPA buffer (150 mM NaCl, $1 \%$ Triton X-100, 0.5\% sodium

135 deoxycholate, $0.1 \%$ SDS, $50 \mathrm{mM}$ Tris, $\mathrm{pH} 8.0$ ) supplemented with $1 \%$ protease inhibitor

136 cocktail (Sigma-Aldrich, Munich, Germany) and 1\% phosphatase inhibitor cocktail II and III

137 (Sigma-Aldrich, P5726, P0044), the whole procedure was performed at $4^{\circ} \mathrm{C}$. Protein

138 concentration was measured by Bradford assay and $20 \mu \mathrm{g}$ of protein was loaded on a $10 \%$

139 polyacrylamide gel after being denatured at $95^{\circ} \mathrm{C}$ for $5 \mathrm{~min}$. After SDS-PAGE, gels were

140 blotted onto a nitrocellulose membrane (GE Healthcare, Buckinghamshire, UK) and later

141 blocked in 5\% milk and probed with the following antibodies: phospo-CREB (1:6000,

142 Millipore \#05-667), total-CREB (1:5000, Cell Signaling, \#4820), $\beta$-Actin (1:1000, Santa Cruz,

143 \#SC-47778) or a-Tubulin (1:40000, Sigma-Aldrich, \#T9026). Antibodies were diluted in 5\%

144 milk in PBS-T (total-CREB, $\alpha$-Tubulin and $\beta$-Actin) or in $5 \%$ bovine serum albumin in PBS-T

145 (phospho-CREB). Next, the membranes were incubated with horseradish peroxidase-

146 conjugated secondary antibodies and later analyzed using a ChemiDoc ${ }^{\mathrm{TM}}$ Imaging System

147 (Bio-Rad). Data is presented as ratio of phosphorylated/total protein normalized internally to

148 each uninfected condition.

$149 \quad$ 2.8. Statistical information. For normally distributed data sets, two-tailed

150 unpaired Student's t test or one-way ANOVA were used to compare two or more groups

151 respectively (significant data is marked with *). Two-tailed Mann-Whitney test was used to

152 compare two distinct groups for non-Gaussian distribution (significant data is marked with \#).

153 Correlation analysis was performed using Pearson correlation coefficient or Spearman

154 correlation for normally distributed or non-parametric data, respectively. The sample size was

155 determined based on similar experiments carried out in the past. All plotted data represent

156 mean \pm SEM. Statistics were performed using GraphPad Prism for Mac OS X, version 8. For

157 behavioral experiments the investigators were blind to group allocation during data collection

158 and analysis. For in vitro experiments no blinding was performed since the outcome was

159 dependent on software analysis and not manual scoring. 


\section{Results}

\subsection{Aging increases Gadd45y expression in the human hippocampus.}

Aberrant gene expression patterns are an evolutionarily conserved hallmark of aging. However, no overall correlation between age-associated gene expression in mice and humans has been detected (Zahn et al., 2007). We asked whether Gadd45y expression in human aged hippocampus would be compromised as observed in mice (Brito et al., 2020).

We analyzed the expression of Gadd45 family members in young and aged human hippocampi as we previously described (21-65 years old) (Temido-Ferreira et al., 2018)

(Figure 1A). We did not find any correlation between age and Gadd45 $\alpha$ expression. Interestingly, we found that hippocampal Gadd $45 \beta$ and Gadd45y levels were increased ( 4.8 and $\sim 8.6$ fold, respectively) as age progressed. This result, together with our previous findings in aged mouse tissue (Brito et al., 2020), suggests that age-related Gadd45 expression changes in the hippocampus may not be conserved in mice and humans.

\subsection{Gadd45y overexpression leads to impairments in spatial recognition}

memory. Next, we sought to model the human aging-associated Gadd45y increase in the mouse hippocampus and determine the cellular and behavioral consequences of neuronal Gadd45y overexpression. Given that previous studies showed a selective function for Gadd45y in memory formation (Brito et al., 2020; Li et al., 2018), we focused on Gadd45y. We stereotaxically delivered a viral vector containing the mouse CamKIla promoter driving the expression of Gadd45y, or GFP as a control, into the dorsal hippocampus (dHPC) of young adult mice (Figure 1B,C). We validated viral expression in the dHPC of injected animals by assessing GFP expression and Gadd45y mRNA levels (Figure S1A-C). Neither group showed anatomical or histological brain abnormalities. Two weeks after stereotaxic surgery, before assessing cognitive function, we performed an open field test (Figure 1C) to verify whether Gadd45y overexpression affects locomotor activity or anxiety-like behavior. Total distance travelled and the percentage of the time spent in the central zone were similar between groups (Figure 1D-F). Next, we assessed long-term memory in the object-place recognition test and contextual fear conditioning. Increasing Gadd45y expression in the 
189 dHPC of young mice impaired preference for the displaced object $24 \mathrm{~h}$ after learning (Figure

190 1G). This impairment was not due to altered habituation patterns during the training trial

191 sessions or altered object exploratory behavior (Figure S1D,E). In contrast, Gadd45yOE

192 mice showed intact long-term memory in contextual fear conditioning (Figure 1H). Both

193 groups presented similar responses to shock administration (Figure S1F).

194 3.3. Gadd45Y overexpression disrupts activity-dependent CREB activation

195 and gene expression. Considering that Gadd45y regulates CREB activity (Brito et al.,

196 2020), we next investigated whether Gadd45y overexpression would impact CREB

197 regulation. First, we assessed whether Gadd45y overexpression in the hippocampus of

198 young adult mice (Figure 2A) affects the levels of phosphorylated CREB in baseline

199 conditions. In agreement with a role for Gadd45y in signaling pathways that regulate CREB

200 activation (Brito et al., 2020), overexpression of Gadd45y resulted in increased levels of

201 phosphorylated CREB (Figure 2B,C). Next using primary hippocampal cultures (Figure

202 S1G,H) we analyzed the phosphorylation of CREB in baseline conditions and in response to

203 increased neuronal activity (Figure 2D). Similar to our in vivo findings (Figure 2A-C),

204 Gadd45y overexpression in primary hippocampal cultures led to increased levels of

205 phosphorylated CREB in basal conditions. Moreover, this effect appeared to blunt a

206 response to neuronal activity; CREB phosphorylation in response to neuronal activity did not

207 reach controls levels (Figure 2E,F). We next assessed the expression of the CREB-

208 dependent genes Arc, FosB, c-Fos, Egr1 and Npas4 (Impey et al., 2004; Rao-Ruiz et al.,

209 2019) in basal conditions and upon neuronal activity (Figure 2G). Hippocampal neuronal

210 cultures infected with rAAV-Gadd45yOE revealed disrupted CREB-dependent gene

211 expression in response to increased neuronal activity compared to control conditions (Figure

$2122 \mathrm{H}-\mathrm{L}$ ). This set of experiments shows that increasing Gadd45y above physiological levels in

213 hippocampal neurons disrupts CREB phosphorylation and gene expression required for

214 memory formation. Taken together, these findings demonstrate that an increase in

215 hippocampal Gadd45y levels disrupts CREB regulation, the expression of memory-related

216 genes and cognitive function. 


\section{Discussion}

219 This study suggested that human aging is associated with increased hippocampal Gadd45y expression. Together with our previous findings (Brito et al., 2020) we showed that

221 bidirectional dysregulation of hippocampal Gadd45y levels in young adult mice negatively

222 impacts cognitive function, CREB regulation and the expression of memory-related genes.

223 Thus, implicating a requirement for tight control of Gadd45y levels in brain function.

224 We observed that mimicking the human aging-related increase in Gadd45y expression in the 225 mouse hippocampus or in dissociated hippocampal neurons, promoted memory deficits and 226 impairments in CREB-dependent gene transcription, respectively. Both the reduction or

227 chronic enhancement of CREB function is known to lead to spatial memory deficits (Li et al., 228 2015; Pittenger et al., 2002; Viosca et al., 2009), as described in studies using CREB229 deficient mutants (Pittenger et al., 2002) or mouse models that express constitutively active

230 forms of CREB such as VP16-CREB (Viosca et al., 2009). Moreover, constitutive CREB

231 activation has been identified as a possible contributing mechanism involved in Alzheimer's

232 disease (Muller et al., 2011). In our study Gadd45y overexpression induced increased levels

233 of phosphorylated CREB in basal conditions both in the mouse hippocampus and in

234 hippocampal cultures. Although this remains to be investigated, the observed increase in

235 Gadd45y expression levels in the aged human hippocampus, may also result in chronic

236 elevations in the activated form of CREB. Our experiments in hippocampal cultures indicate

237 that increased levels of phosphorylated CREB already in basal conditions impair a response

238 to neuronal activity and result in deficits in the expression of plasticity-related genes. Thus,

239 providing a possible mechanism for impaired cognitive function in conditions of elevated

240 Gadd45y expression like in our model and possibly in the hippocampus of aged individuals.

241 Together with our previous findings, which showed that Gadd45y knockdown leads to

242 impairments in CREB activation and associated gene expression (Brito et al., 2020), this

243 data suggests that proper cellular function requires the tight regulation of Gadd $45 y$ levels. 
244 These findings are in agreement with another study showing that either Gadd $45 y$ loss- or

245 gain-of-function disrupts neural development (Kaufmann and Niehrs, 2011).

246 The deficits in memory were task-specific; young adult mice expressing Gadd45y above

247 physiological levels presented selective long-term memory impairments in object place-

248 recognition memory but not in contextual fear conditioning. Intriguingly, similar results have

249 been found in aged mice and humans. It has been described that aged mice (Kennard and

250 Woodruff-Pak, 2011) and humans (Battaglia et al., 2018; Foster et al., 2012; Leal and Yassa,

2512015 ) are more likely to display deficits in forms of recognition memory than in contextual

252 fear conditioning. The reasons for the selective impairment may be attributed to the

253 characteristics of the tasks; despite hippocampal dysfunction in response to aging or

254 Gadd45y overexpression, mice may still be able to form and store the association between a

255 highly salient stimulus (novel context) and a foot-shock. Similar findings have been described

256 for other models of impaired hippocampal function. Namely, in a mouse model of Rett

257 syndrome (Gulmez Karaca et al., 2018) and Alzheimer's disease (Corcoran et al., 2002). In

258 the later, contextual fear conditioning impairments were only present when the salience of

259 the context was reduced.

260 Aberrant gene transcription patterns occur as a consequence of aging in the hippocampus

261 (Burger, 2010; lanov et al., 2017; Verbitsky et al., 2004). These changes do not overly

262 correlate across species (Bishop et al., 2010; Loerch et al., 2008; Zahn et al., 2007), thus

263 limiting the translational potential of animal models. Studies comparing cross-species

264 alterations in gene expression generally focus on shared changes. The similar

265 consequences of bidirectional dysregulation of Gadd45y expression levels suggest that this

266 approach may neglect functionally relevant and seemingly disparate age-associated

267 transcription changes. Using in vivo and in vitro models we show that hippocampal levels of

268 Gadd45y are tightly regulated and that either a decrease (Brito et al., 2020) or an increase in

269 Gadd45y can dysregulate plasticity-associated gene expression and cause cognitive

270 impairments. Notably, previous studies that performed transcriptomic analysis of human

271 hippocampal tissue throughout adulthood also suggested an age-associated increase in 
bioRxiv preprint doi: https://doi.org/10.1101/2020.01.12.903112; this version posted June 29, 2020. The copyright holder for this preprint (which was not certified by peer review) is the author/funder, who has granted bioRxiv a license to display the preprint in perpetuity. It is made available under aCC-BY-NC-ND 4.0 International license.

272 Gadd45y expression (Kang et al., 2011; Pletikos et al., 2014). Accordingly, our findings

273 illustrate a scenario in which diverging age-related transcriptional programs in mice and

274 humans result in converging phenotypes.

275 Taken together, our results demonstrate the requirement for tight control of Gadd45y levels

276 in memory formation and further implicate Gadd45y as a molecular candidate that may

277 underlie cognitive impairments in aging-associated pathological conditions.

278

279 Acknowledgements

280 We thank I. Bu $\square$ nzli-Ehret for the preparation of primary hippocampal cultures and Stephanie

281 Zeuch for comments to the manuscript. This work was supported by the Deutsche

282 Forschungsgemeinschaft (DFG) [SFB 1134 (C01), OL 437/1 to A.M.M.O.], by Chica and

283 Heinz Schaller foundation [fellowship to A.M.M.O.] and by Santa Casa da Misericórdia

284 Mantero Belard Award [MB-07-2018 to L.V.L.].

285

286 Disclosure statement

287 The authors declare no conflict of interest.

References

Aigbogun, M.S., Stellhorn, R., Krasa, H., Kostic, D., 2017. Severity of memory impairment in the elderly: Association with health care resource use and functional limitations in the United States. Alzheimer's \& dementia (Amsterdam, Netherlands) 8, 51-59.

Battaglia, S., Garofalo, S., di Pellegrino, G., 2018. Context-dependent extinction of threat memories: influences of healthy aging. Scientific reports 8(1), 12592.

Bishop, N.A., Lu, T., Yankner, B.A., 2010. Neural mechanisms of ageing and cognitive decline. Nature 464(7288), 529-535.

Brito, D.V.C., Kupke, J., Gulmez Karaca, K., Zeuch, B., Oliveira, A.M.M., 2020. Mimicking age-associated Gadd45 $\gamma$ dysregulation results in memory impairments in young adult mice. The Journal of Neuroscience, 1621-1619.

Burger, C., 2010. Region-specific genetic alterations in the aging hippocampus: implications for cognitive aging. Front Aging Neurosci 2, 140.

Corcoran, K.A., Lu, Y., Turner, R.S., Maren, S., 2002. Overexpression of hAPPswe impairs rewarded alternation and contextual fear conditioning in a transgenic mouse model of Alzheimer's disease. Learning \& memory (Cold Spring Harbor, N.Y.) 9(5), 243-252.

Foster, T.C., Defazio, R.A., Bizon, J.L., 2012. Characterizing cognitive aging of spatial and contextual memory in animal models. Front Aging Neurosci 4, 12.

Gulmez Karaca, K., Brito, D.V.C., Zeuch, B., Oliveira, A.M.M., 2018. Adult hippocampal MeCP2 preserves the genomic responsiveness to learning required for long-term memory formation. Neurobiology of learning and memory $149,84-97$. 
310

311

312

313

314

315

316

317

318

319

320

321

322

323

324

325

326

327

328

329

330

331

332

333

334

335

336

337

338

339

340

341

342

343

344

345

346

347

348

349

350

351

352

353

354

355

356

357

358

359

360

361

362

363

364

365

366

367

368

369

370

371

Gulmez Karaca, K., Kupke, J., Brito, D.V.C., Zeuch, B., Thome, C., Weichenhan, D., Lutsik, P., Plass, C., Oliveira, A.M.M., 2020. Neuronal ensemble-specific DNA methylation strengthens engram stability. Nature communications 11(1), 639.

Ianov, L., De Both, M., Chawla, M.K., Rani, A., Kennedy, A.J., Piras, I., Day, J.J., Siniard, A., Kumar, A., Sweatt, J.D., Barnes, C.A., Huentelman, M.J., Foster, T.C., 2017. Hippocampal Transcriptomic Profiles: Subfield Vulnerability to Age and Cognitive Impairment. Front Aging Neurosci 9, 383.

Impey, S., McCorkle, S.R., Cha-Molstad, H., Dwyer, J.M., Yochum, G.S., Boss, J.M., McWeeney, S., Dunn, J.J., Mandel, G., Goodman, R.H., 2004. Defining the CREB regulon: a genome-wide analysis of transcription factor regulatory regions. Cell 119(7), 1041-1054.

Kang, H.J., Kawasawa, Y.I., Cheng, F., Zhu, Y., Xu, X., Li, M., Sousa, A.M., Pletikos, M., Meyer, K.A., Sedmak, G., Guennel, T., Shin, Y., Johnson, M.B., Krsnik, Z., Mayer, S., Fertuzinhos, S., Umlauf, S., Lisgo, S.N., Vortmeyer, A., Weinberger, D.R., Mane, S., Hyde, T.M., Huttner, A., Reimers, M., Kleinman, J.E., Sestan, N., 2011. Spatio-temporal transcriptome of the human brain. Nature 478(7370), 483-489.

Kaufmann, L.T., Niehrs, C., 2011. Gadd45a and Gadd45g regulate neural development and exit from pluripotency in Xenopus. Mechanisms of development 128(7-10), 401-411.

Kennard, J.A., Woodruff-Pak, D.S., 2011. Age sensitivity of behavioral tests and brain substrates of normal aging in mice. Front Aging Neurosci 3, 9.

Leal, S.L., Yassa, M.A., 2015. Neurocognitive Aging and the Hippocampus across Species. Trends in neurosciences 38(12), 800-812.

Li, P., Rial, D., Canas, P.M., Yoo, J.H., Li, W., Zhou, X., Wang, Y., van Westen, G.J., Payen, M.P., Augusto, E., Goncalves, N., Tome, A.R., Li, Z., Wu, Z., Hou, X., Zhou, Y., A, P.I., Boyden, E.S., Cunha, R.A., Qu, J., Chen, J.F., 2015. Optogenetic activation of intracellular adenosine A2A receptor signaling in the hippocampus is sufficient to trigger CREB phosphorylation and impair memory. Molecular psychiatry 20(11), 1481.

Li, X., Marshall, P.R., Leighton, L.J., Zajaczkowski, E.L., Wang, Z., Madugalle, S.U., Yin, J., Bredy, T.W., Wei, W., 2018. The DNA repair associated protein Gadd45 regulates the temporal coding of immediate early gene expression within the prelimbic prefrontal cortex and is required for the consolidation of associative fear memory. J Neurosci.

Loerch, P.M., Lu, T., Dakin, K.A., Vann, J.M., Isaacs, A., Geula, C., Wang, J., Pan, Y., Gabuzda, D.H., Li, C., Prolla, T.A., Yankner, B.A., 2008. Evolution of the aging brain transcriptome and synaptic regulation. PloS one 3(10), e3329.

Muller, M., Cardenas, C., Mei, L., Cheung, K.H., Foskett, J.K., 2011. Constitutive cAMP response element binding protein (CREB) activation by Alzheimer's disease presenilin-driven inositol trisphosphate receptor (InsP3R) Ca2+ signaling. Proceedings of the National Academy of Sciences of the United States of America 108(32), 13293-13298.

Oliveira, A.M., Hemstedt, T.J., Bading, H., 2012. Rescue of aging-associated decline in Dnmt3a2 expression restores cognitive abilities. Nat Neurosci 15(8), 1111-1113.

Oliveira, A.M., Hemstedt, T.J., Freitag, H.E., Bading, H., 2016. Dnmt3a2: a hub for enhancing cognitive functions. Mol Psychiatry 21(8), 1130-1136.

Pittenger, C., Huang, Y.Y., Paletzki, R.F., Bourtchouladze, R., Scanlin, H., Vronskaya, S., Kandel, E.R., 2002. Reversible inhibition of CREB/ATF transcription factors in region CA1 of the dorsal hippocampus disrupts hippocampus-dependent spatial memory. Neuron 34(3), 447-462.

Pletikos, M., Sousa, A.M., Sedmak, G., Meyer, K.A., Zhu, Y., Cheng, F., Li, M., Kawasawa, Y.I., Sestan, N., 2014. Temporal specification and bilaterality of human neocortical topographic gene expression. Neuron 81(2), 321-332.

Pliassova, A., Canas, P.M., Xavier, A.C., da Silva, B.S., Cunha, R.A., Agostinho, P., 2016. Age-Related Changes in the Synaptic Density of Amyloid-beta Protein Precursor and Secretases in the Human Cerebral Cortex. J Alzheimers Dis 52(4), 1209-1214.

Rao-Ruiz, P., Couey, J.J., Marcelo, I.M., Bouwkamp, C.G., Slump, D.E., Matos, M.R., van der Loo, R.J., Martins, G.J., van den Hout, M., van, I.W.F., Costa, R.M., van den Oever, M.C., Kushner, S.A., 2019. Engram-specific transcriptome profiling of contextual memory consolidation. Nature communications 10(1), 2232.

Schindelin, J., Arganda-Carreras, I., Frise, E., Kaynig, V., Longair, M., Pietzsch, T., Preibisch, S., Rueden, C., Saalfeld, S., Schmid, B., Tinevez, J.Y., White, D.J., Hartenstein, V., Eliceiri, K., Tomancak, P., Cardona, A., 2012. Fiji: an open-source platform for biological-image analysis. Nature methods 9(7), 676-682.

Singh-Manoux, A., Kivimaki, M., Glymour, M.M., Elbaz, A., Berr, C., Ebmeier, K.P., Ferrie, J.E., Dugravot, A., 2012. Timing of onset of cognitive decline: results from Whitehall II prospective cohort study. BMJ 344, d7622.

Stefanelli, G., Azam, A.B., Walters, B.J., Brimble, M.A., Gettens, C.P., Bouchard-Cannon, P., Cheng, H.M., Davidoff, A.M., Narkaj, K., Day, J.J., Kennedy, A.J., Zovkic, I.B., 2018. Learning and Age-Related Changes in Genome-wide H2A.Z Binding in the Mouse Hippocampus. Cell reports 22(5), 1124-1131. 
372

373

374

375

376

377

378

379

380

381

382

383

384

385

386

387

388

389

390

391

392

393

394

395

396

397

398

399

400

401

402

403

404

405

406

407

Temido-Ferreira, M., Ferreira, D.G., Batalha, V.L., Marques-Morgado, I., Coelho, J.E., Pereira, P., Gomes, R., Pinto, A., Carvalho, S., Canas, P.M., Cuvelier, L., Buee-Scherrer, V., Faivre, E., Baqi, Y., Muller, C.E., Pimentel, J., Schiffmann, S.N., Buee, L., Bader, M., Outeiro, T.F., Blum, D., Cunha, R.A., Marie, H., Pousinha, P.A., Lopes, L.V., 2018. Age-related shift in LTD is dependent on neuronal adenosine A2A receptors interplay with mGluR5 and NMDA receptors. Molecular psychiatry.

Verbitsky, M., Yonan, A.L., Malleret, G., Kandel, E.R., Gilliam, T.C., Pavlidis, P., 2004. Altered hippocampal transcript profile accompanies an age-related spatial memory deficit in mice. Learning \& memory (Cold Spring Harbor, N.Y.) 11(3), 253-260.

Viosca, J., Malleret, G., Bourtchouladze, R., Benito, E., Vronskava, S., Kandel, E.R., Barco, A., 2009. Chronic enhancement of CREB activity in the hippocampus interferes with the retrieval of spatial information. Learning \& memory (Cold Spring Harbor, N.Y.) 16(3), 198-209.

Zahn, J.M., Poosala, S., Owen, A.B., Ingram, D.K., Lustig, A., Carter, A., Weeraratna, A.T., Taub, D.D., Gorospe, M., Mazan-Mamczarz, K., Lakatta, E.G., Boheler, K.R., Xu, X., Mattson, M.P., Falco, G., Ko, M.S., Schlessinger, D., Firman, J., Kummerfeld, S.K., Wood, W.H., 3rd, Zonderman, A.B., Kim, S.K., Becker, K.G., 2007. AGEMAP: a gene expression database for aging in mice. PLoS Genet 3(11), e201.

\section{Figure legends}

Figure 1. Human hippocampal Gadd45y expression is increased during aging and overexpressing Gadd45y in the mouse hippocampus impairs object location memory. A)

Correlational analysis between the expression of Gadd45 $\alpha$, Gadd45 $\beta$ and Gadd $45 \gamma$ in

human postmortem hippocampal tissue and donors' age $(\mathrm{N}=6)$. Correlation analysis was

performed using Pearson correlation coefficient or Spearman correlation. B) Schematic

representation of the viral constructs used. The viral vector contains a CamKIl $\alpha$ promoter

driving Gadd45y overexpression (Gadd45yOE) or GFP as a control (GFP). C) Schematic

representation of the experimental design for behavioral tests. D) Representative exploration

patterns of all groups during open field test. E) Locomotion analysis of the different groups

measured as the total distance travelled during the open field test $(\mathrm{N}=8-9)$. F) Anxiety-like

behavior analysis measured as percentage of time spent in the center of the arena during the

open field test ( $\mathrm{N}=8-9)$. G) $24 \mathrm{~h}$ object location memory test of young adult mice expressing

GFP or Gadd45yOE in the dHPC (N=13-15). Dashed line represents equal preference for

either object (chance preference). H) 24h contextual fear memory test of young adult mice

expressing GFP or Gadd45yOE in the dHPC (N=9). ns: not significant; ${ }^{* *} \mathrm{p}<0.01$ by two-tailed

unpaired Student's t-test. Error bars represent SEM.

Figure 2. Increased Gadd45y expression dysregulates hippocampal CREB phosphorylation

and activity-dependent gene expression. A) Schematic representation of the experimental 
408 design for western blot analysis of CREB activation in vivo. B) Representative immunoblot

409 scans of hippocampal tissue from young adult mice infected with rAAVs expressing GFP or

410 Gadd45yOE using phosphospecific (pCREB) and total CREB (tCREB) antibodies. C)

411 Immunoblot quantification shown as ratios of phosphorylated/total protein normalized to GFP

412 control (N=5-6). D) Schematic representation of the experimental design for western blot

413 analysis of CREB activation in mouse hippocampal cultures. E) Representative immunoblot

414 scans of hippocampal cultures infected with rAAV expressing GFP or Gadd45yOE using

415 phosphospecific and total CREB antibodies. F) Immunoblot quantification shown as ratios of

416 phosphorylated/total protein normalized to uninfected control in baseline or bicuculline-

417 treated conditions (left and middle graphs) or pCREB fold increase (bicuculline/baseline) for

418 each condition and normalized to uninfected control (right graph) ( $\mathrm{N}=7-8$ independent cell

419 preparations). G) Schematic representation of the experimental design for qRT-PCR analysis

420 of the expression of CREB-dependent genes ( $N=5-6$ independent cell preparations) H) Arc,

421 I) c-Fos J) FosB K) Egr1 and L) Npas4 in hippocampal cultures. Hippocampal cultures were

422 harvested at baseline conditions or after $2 h, 4 h$, or $6 h$ of bicuculline treatment. ${ }^{*} p<0.05$,

$423{ }^{* *} p<0.01,{ }^{* *} p<0.001$ and ${ }^{* * * *} p<0.0001$ by two-tailed Student's t-test. ns: not significant. Error

424 bars represent SEM.

425

426 
A

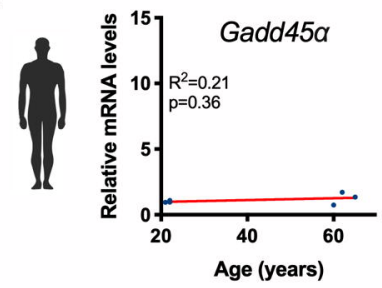

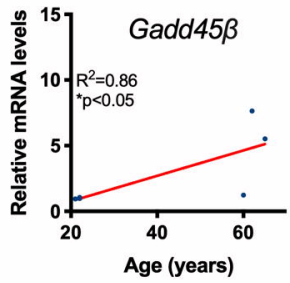

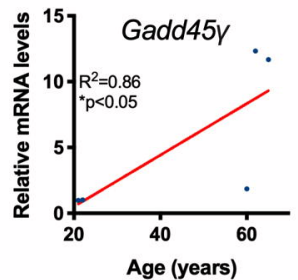

B

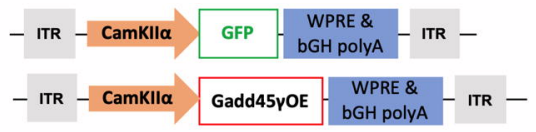

C

Bilateral rAAV injections
into the dHPC into the dHPC
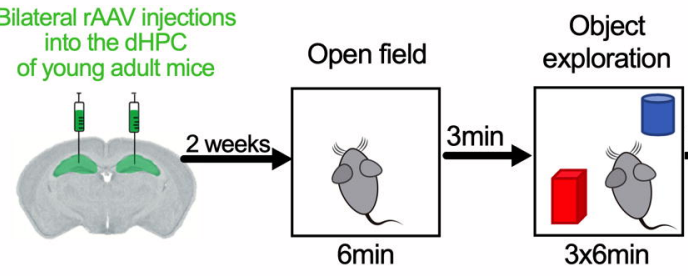

Object location

$$
\text { test }
$$
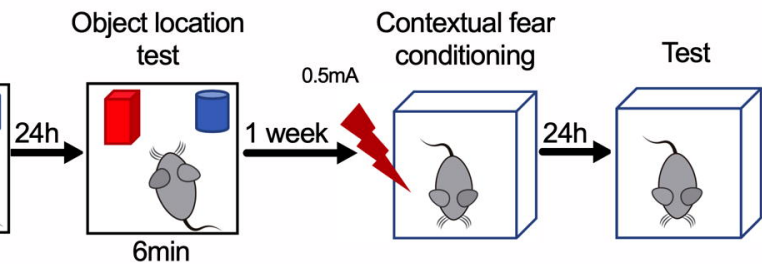

E

F

G Object location test

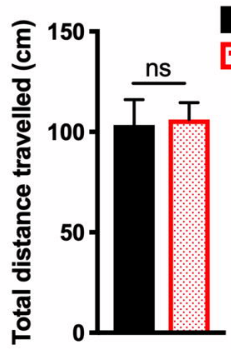

GFP

G Gadd45YOE

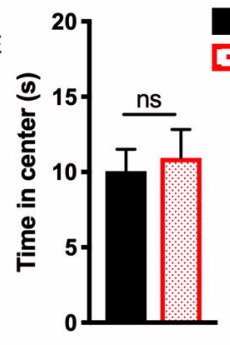

GFP

Gadd45yOE

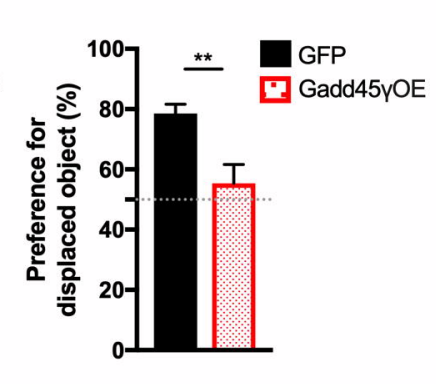

H contextual fear

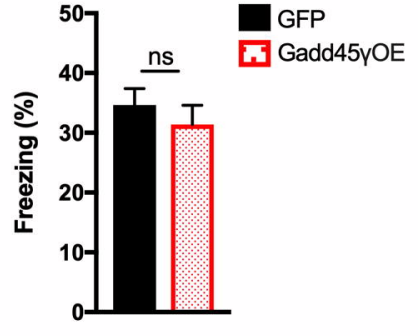

GFP

Gadd45yOE 
